\section{Colonic crypt cell proliferation state assessed by whole crypt microdissection in sporadic neoplasia and familial adenomatous polyposis}

\author{
S J Mills, J C Mathers, P D Chapman, J Burn, A Gunn
}

together, between sporadic disease and FAP, and between adenoma and carcinoma subjects at each of the four colonic sites. Post hoc comparison by $t$ test showed significantly greater CCPS for FAP compared with controls $(\mathbf{p}<0.001)$ and for sporadic cancer versus controls $(\mathbf{p}=0.04)$.

Conclusions-Whole crypt microdissection and mitosis counting is a reliable, reproducible, and robust technique for assessing CCPS in the human colon. CCPS is unaffected by age but increases from the distal to the proximal colon. CCPS is increased if a sporadic cancer is present and markedly increased in FAP. However, the precise relation of an increased CCPS to the neoplastic process remains uncertain.

(Gut 2001;48:41-46)

Subjects-Mucosal samples were obtained at colectomy or colonoscopy from 107 individuals ( 24 controls, 23 sporadic adenoma patients, 31 sporadic carcinoma patients, and 29 patients with familial adenomatous polyposis (FAP)).

Methods-Mucosal specimens were hydrated, hydrolysed, and small groups of crypts separated from the main specimen under a dissecting microscope. The total number of mitoses/crypt were counted by one observer for each of 10 complete crypts.

Results-Validation work established that whole crypt mitoses counts were reliable and reproducible. There was no relation between age and mean mitoses/crypt (Pearson correlation coefficient -0.1). The CCPS count was higher for males than for females (difference in means 2.8 (95\% confidence interval $0.80-4.66)$ ) among controls but there was no gender difference in the three disease groups. For all disease groups and controls, the crypt mitotic count showed a significant linear increase $(p=0.004)$ from the rectum to the caecum. Biopsies from within $5 \mathrm{~cm}$ of the macroscopic margin of a carcinoma (near) gave a mean mitosis count of $\mathbf{1 2 . 6}$ while those from more than $10 \mathrm{~cm}$ (far) were lower but not significantly so $(p=0.12)$ with a count of 9.0 . The mean mitoses/crypt were similar for the controls and adenomas (5.6 and 4.7, respectively) but greater for the cancers and especially for FAP (8.3 and 14.2, respectively). Statistical analysis confirmed that there were significant differences $(p<0.05)$ between controls and all disease groups
Keywords: colonic crypt; cell proliferation; familial adenomatous polyposis; colorectal cancer

Homeostasis and health in adulthood requires an exquisite balance between cell proliferation and cell loss by apoptosis and other routes. Failure to match cell birth with cell death results in loss of tissue integrity, for example in ulceration, or in abnormal growth-that is, tumorigenesis. There has been considerable work in recent years relating proliferative activity in human tissues to the risk of malignant transformation. Several workers have found known to be at risk of colorectal carcinoma, ${ }^{1}$ particularly patients with familial adenomatous polyposis (FAP). ${ }^{2}$ Controversy persists however as to the biological relevance of these findings, not least as a result of the methodological limitations inherent in using indirect indices of proliferation necessitated by studies in human subjects. Relatively little has been published on normal crypt cell proliferation in the human colon. It may be influenced by many factors, including sampling methodology, age, ${ }^{3}$ sex, diet, ${ }^{4}$ bowel preparation, and variation at different sites throughout the large intestine $\mathrm{e}^{5}$ as well as by the "neoplastic risk" of the individual. ${ }^{6}$ Abnormalities of crypt proliferative activity could represent an early field change with neoplastic potential or could be a reactive phenomenon to the presence of neoplastic disease. Clarification of the significance of

Abbreviations used in this paper: CCPS, crypt cell proliferative state; FAP, familial adenomatous polyposis; HNPCC, hereditary non-polyposis colorectal cancer. abnormal indices of proliferation in groups 
abnormal proliferative activity would have important consequences regarding its usefulness as a biomarker of neoplastic risk or as a surrogate endpoint for chemoprevention trials.

Methods used to assess cellular proliferation can be divided broadly into dynamic techniques which reflect proliferative rate and static techniques that reflect proliferative state. Proliferative rate is the more biologically relevant measurement but its assessment requires either systemic administration of cytotoxic drugs or in vitro incubation of tissue- - the former is not applicable in human subjects and the latter is subject to artefacts introduced by removal of the tissue from in vivo homeostatic influences. Mitosis counting following whole crypt microdissection is a measure of crypt cell proliferative state (CCPS) but addresses many of the methodological criticisms levelled at immunohistochemical methods. A particular advance is the ability to express results with the whole crypt as the denominator, avoiding the shortcomings of longitudinal crypt sections. ${ }^{78}$ The reliability of this method was tested on a small group of control subjects and then applied to investigate the impact of normal biological variables and neoplastic risk on the CCPS throughout the colon using 348 samples from 107 patients.

\section{Methods}

Ethics committee approval was obtained from all health districts in the northern region of England. Samples of colonic mucosa from macroscopically normal colon were collected either at colonoscopy or from the specimen at colectomy. Bowel preparation for colonoscopy consisted of $50 \mathrm{ml}$ of senna and a full sachet of picolax the day prior to colonoscopy, and half a sachet of picolax on the morning of the examination. For flexible sigmoidoscopy, senna was omitted. Where possible, biopsies were taken from four sites: right colon, left colon, sigmoid colon, and rectum. The majority of colonoscopies were performed by a single experienced endoscopist (AG) with an Olympus CF or FS biopsy forceps and an Olympus IT200L or Olympus 200S videoendoscope. Biopsies were immediately placed in fixative (ethanol:acetic acid 75:25) and refrigerated. At the time of analysis, they were hydrated in successive aqueous washes $(50 \%$ ethanol for 10 minutes, $25 \%$ ethanol for 10 minutes) and then hydrolysed in $1 \mathrm{M}$ of $\mathrm{HCl}$ for 10 minutes at $60^{\circ} \mathrm{C}$ before being stained in Schiff's reagent for 60 minutes. Stained samples of tissue were placed in a drop of acetic acid on a slide under a dissecting microscope $(\times 4)$ and small groups of crypts teased apart with hypodermic needles. Under higher power $(\times 40), 10 \mathrm{com}-$ plete crypts were assessed for each specimen. By focusing up and down through the width of the crypt, a total mitotic count for each crypt was obtained. ${ }^{9}$ All counting was performed by a single blinded observer (SJM) using strict criteria for inclusion of a mitotic fig. Only figs in late prophase, metaphase, anaphase, or early telophase were counted. Exclusion of early prophase and late telophase aided demarcation

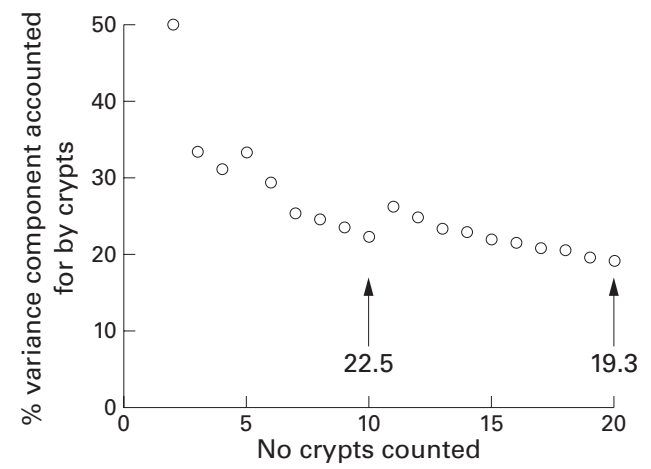

Figure 1 Repeated analysis of variance for differing numbers of crypts counted for seven control subjects.

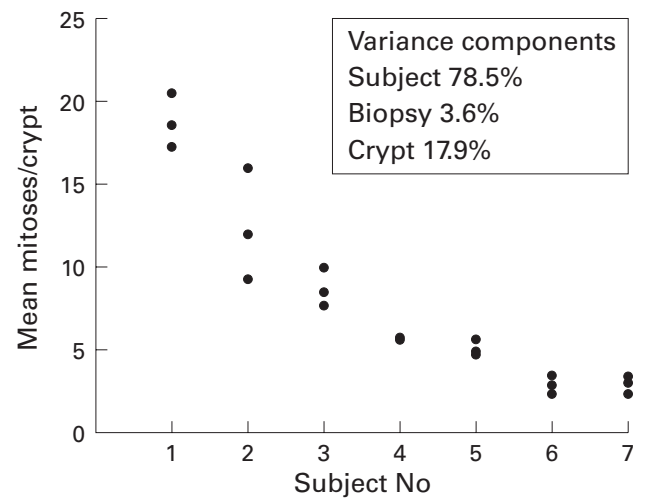

Figure 2 Mean mitoses/crypt and variance components for three biopsies from each rectum of seven control subjects.

between mitotic and non-mitotic figs which helped to give valid and reproducible results.

\section{VALIDATION}

Number of crypts counted per biopsy (fig 1)

The measurement error estimated as the pooled standard error of the mean for the counts of 20 crypts from single rectal biopsies from seven control subjects was 0.58 for an overall mean of 7.5 mitoses/crypt. The percentage variance component accounted for by the intercrypt or within individual variance decreased rapidly from two to 10 crypts. Increasing the number of crypts counted to 20 had little effect. The remainder of the study was therefore performed on counts of 10 crypts/ biopsy.

The value of a single biopsy as representative of the rectum (fig 2)

Analysis of variance for the three rectal biopsies from the seven control subjects showed that most $(78.5 \%)$ of the variance was due to differences between subjects and $3.6 \%$ to between biopsy variation, establishing that a single biopsy is representative of that part of the large bowel for a given individual.

\section{SUBJECTS}

The control group were either patients referred for colonoscopy for symptoms of rectal bleeding or change in bowel habit in whom colonoscopy was macroscopically normal (colonoscopy negative controls) or members of FAP pedigrees subsequently found to be gene negative on DNA analysis or to have a $<1 \%$ risk 
Table 1 Patient characteristics

\begin{tabular}{lllll}
\hline & Control & $\begin{array}{l}\text { Sporadic } \\
\text { adenoma }\end{array}$ & $\begin{array}{l}\text { Sporadic } \\
\text { carcinoma }\end{array}$ & FAP \\
\hline $\mathrm{n}$ & $24^{1}$ & 23 & $31^{2}$ & $29^{3}$ \\
Age (y) (range) & $48(20-74)$ & $68(54-82)$ & $67(35-81)$ & $29(14-64)$ \\
Sex (M:F) & $15: 9$ & $16: 7$ & $20: 11$ & $13: 16$ \\
\hline
\end{tabular}

Subgroups: ${ }^{1} 12$ colonoscopy negative and 12 gene negative controls; ${ }^{2} 20$ concurrent and 11 previous carcinomas; ${ }^{3} 22$ intact and seven postcolectomy patients with familial adenomatous polyposis (FAP).

Table 2 Mean mitoses/crypt by colonic site and disease group

\begin{tabular}{lllll}
\hline & Control & $\begin{array}{l}\text { Sporadic } \\
\text { adenoma }\end{array}$ & $\begin{array}{l}\text { Sporadic } \\
\text { carcinoma }\end{array}$ & FAP \\
\hline Rectum & & & & \\
Number & 24 & 22 & 17 & 28 \\
Mean mitoses/crypt & 5.6 & 4.7 & 8.3 & 14.2 \\
$\quad$ SEM & 0.6 & 0.49 & 1.05 & 0.85 \\
Sigmoid colon & & & & \\
Number & 23 & 23 & 19 & 15 \\
Mean mitoses/crypt & 5.7 & 4.7 & 9.0 & 15.5 \\
SEM & 0.63 & 0.48 & 0.96 & 1.68 \\
Left colon & & & & \\
Number & 19 & 23 & 17 & 15 \\
Mean mitoses/crypt & 6.6 & 5.3 & 10.3 & 17.5 \\
SEM & 0.74 & 0.40 & 1.66 & 1.66 \\
Right colon & 18 & & & \\
Number & 8.8 & 7.0 & 20 & 15 \\
Mean mitoses/crypt & 0.86 & 0.45 & 12.4 & 19.9 \\
SEM & $\mathrm{y}=0.984 \mathrm{x}+4.11$ & $\mathrm{y}=0.735 \mathrm{x}+3.56$ & $\mathrm{y}=1.41 \mathrm{x}+6.50$ & $\mathrm{y}=1.87 \mathrm{x}+12.1$ \\
Regression equation & $\mathrm{p}=0.002$ & $\mathrm{p}=0.001$ & $\mathrm{p}=0.004$ & $\mathrm{p}=0.002$ \\
& $r^{2}=10.7 \%$ & $r^{2}=12.6 \%$ & $r^{2}=11.1 \%$ & $r^{2}=12.6 \%$ \\
\hline
\end{tabular}

on the basis of screening (gene negative controls). The sporadic adenoma patients were either attending for surveillance colonoscopy having previously been diagnosed with an adenomatous polyp(s) or were found to have an adenomatous polyp at diagnostic colonoscopy. Sporadic carcinoma patients were attending for surveillance colonoscopy following colonic resection for carcinoma, were diagnosed at colonoscopy with carcinoma, or were undergoing colonic resection for carcinoma. The specimens from patients with FAP were obtained at preoperative colonoscopy, postoperative sigmoidoscopy, or at the time of colectomy. All patients with FAP had adenomas but none had a carcinoma. Demographic data for each patient were collected prospectively at the time of specimen collection and patients were interviewed to document any family history of colorectal carcinoma.

Sets of mucosal specimens were obtained from 116 patients (including repeat biopsies of some patients), 31 at the time of colorectal resection and 108 at endoscopy (table 1). Nine of the patients were difficult to classify as regards neoplastic risk and were therefore excluded from further analysis (five from FAP pedigrees of uncertain gene status, two from a possible hereditary non-polyposis colorectal cancer (HNPCC) pedigree, one with a possible family history of colorectal carcinoma, and one with Peutz-Jeghers syndrome). Thus the remaining subjects were 24 controls (12 gene negative controls, 12 colonoscopy negative controls), 23 sporadic adenoma patients, 31 sporadic carcinoma patients (20 concurrent and 11 previous carcinomas), and 29 APC gene mutation carriers ( 22 with an intact colon and seven postcolectomy). In 11 patients with sporadic carcinoma, specimens were obtained both near $(<5 \mathrm{~cm})$ and far $(>10 \mathrm{~cm})$ from the tumour. In nine of the FAP patients, samples were obtained on more than one occasion.

STATISTICAL METHODS

Mitosis counts of the rectum, pooled from each of seven control subjects, were analysed on a crypt by crypt basis for total numbers of crypts counted from 1 to 20 by repeated analysis of variance to determine the variance component contributed by within individual intercrypt variance. Comparison between two groups of observations (CCPS for males and females and subgroup comparisons) were made using a two sample $t$ test, and paired data comparisons (stability of CCPS over time) using a paired $t$ test. Comparison between disease groups was performed by one way analysis of variance in which the three degrees of freedom (df) and accompanying sums of squares associated with between disease group differences were partitioned into single df orthogonal contrasts as follows: contrast 1, control versus disease groups (sporadic adenomas and sporadic carcinomas and FAP); contrast 2, sporadic disease (adenomas and carcinomas) versus FAP; contrast 3, sporadic adenoma versus sporadic carcinoma. In each case, the contrast mean square was compared with the between individuals within disease groups mean square using an $\mathrm{F}$ test. To test the effect of colonic site, regression analysis was performed separately for each disease group, with values of $1,2,3$, and 4 allocated to the rectum, sigmoid, and left and right colon, respectively. A post hoc comparison was made of controls versus FAP and controls versus sporadic cancer using a two sample $t$ test.

\section{Results}

EFFECT OF AGE AND SEX ON MITOTIC COUNT

Rectal biopsies from 24 controls (mean age 47.8 years (range $20-74$ )) were examined for the effects of age and sex. There was no relation between age and mean mitoses/crypt (Pearson correlation coefficient -0.1) (table 2). CCPS count was higher for males than for females (M:F 6.7:3.9; $\mathrm{p}=0.008$ ) among controls but there were no gender differences in the three disease groups.

\section{WITHIN GROUPS COMPARISONS}

For obvious reasons, most of the control and adenoma specimens were obtained at endoscopy but a comparison of specimens obtained endoscopically compared with those obtained at colectomy for the carcinoma and FAP groups combined showed no significant difference in CCPS (11.5 and 13.0, respectively; $\mathrm{p}=0.48$ ). Although there was heterogeneity within the colorectal cancer and FAP groups, there was no statistically significant difference between the subgroups concurrent versus previous carcinoma $(p=0.57)$ and intact colon versus postcolectomy for the FAP group $(\mathrm{p}=0.22)$. 


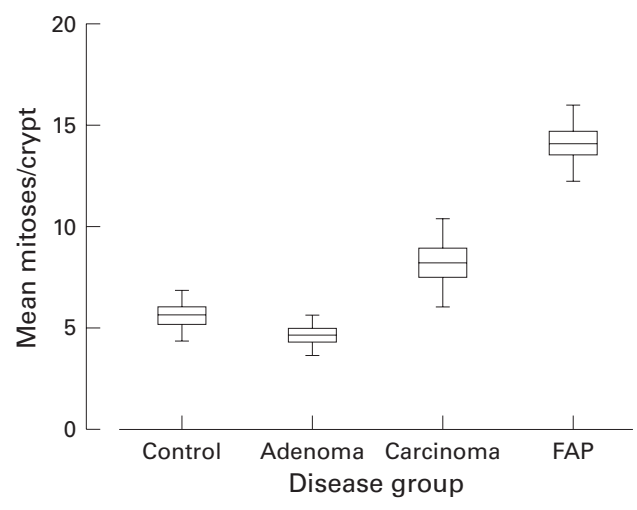

Figure 3 Mean mitoses per crypt of rectal biopsies by disease group with means (bar), 25-75\% percentiles (boxes), and $95 \%$ confidence limits (whiskers). FAP, familial adenomatous polyposis.

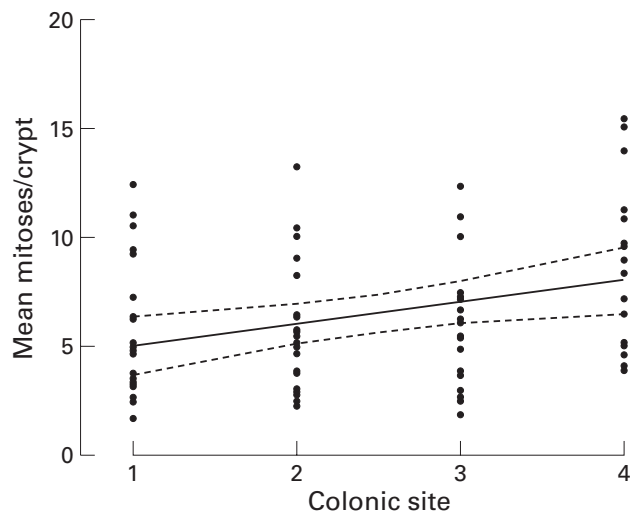

Figure 4 Regression analyses for biopsies from controls for the four colonic sites (1, rectum; 2, sigmoid; 3, left colon; 4, right colon) with $95 \%$ confidence limits.

EFFECT OF NEOPLASTIC RISK AND COLONIC SITE There were differences between the neoplastic risk groups. Mean mitoses/crypt were similar for controls and adenomas (5.6 and 4.7, respectively) but greater for the cancers and FAP (8.3 and 14.2, respectively) (table 2). Statistical analysis confirmed that there were significant differences $(p<0.01)$ between controls and all disease groups together, between sporadic disease and FAP, and between adenoma and carcinoma subjects at each of the four colonic sites (fig 3). For all disease groups and controls, the crypt mitotic count showed a significant linear increase $(p=0.004)$ from the rectum to the caecum (fig 4). Post hoc comparison by $t$ test showed a significantly greater CCPS for FAP compared with controls $(\mathrm{p}<0.001)$ and for sporadic cancer versus controls $(\mathrm{p}=0.04)$.

The effect of proximity of the mucosal specimen to the tumour was investigated for 11 of the sporadic carcinoma colectomies. Biopsies from within $5 \mathrm{~cm}$ of the macroscopic tumour margin (near) gave a mean mitosis score of 12.6 while those from more than $10 \mathrm{~cm}$ (far) were lower with a score of $9.0(p=0.12)$.

Finally, serial rectal biopsies over a period of 4-16 months were obtained from nine FAP patients. Four of these individuals were postcolectomy and two had an intact colon throughout the study. The remaining three underwent colectomy and ileorectal anastomo- sis during the study. The mean mitoses/crypt decreased for two of these patients after ileorectal anastomosis but returned to precolectomy levels in the one individual from whom a further specimen was obtained. The observations after colectomy were excluded to rule out any effect of surgery. In the remaining seven pairs of observations, there was no significant difference between specimens obtained a mean of 10.3 (4-16) months apart $(\mathrm{p}=0.81)$.

\section{Discussion}

The morphological changes involved in the progression from normal mucosa to colorectal adenoma and then to carcinoma have received renewed attention following the identification of oncogenic and tumour suppressor genes associated with the development of colorectal cancer. ${ }^{1011}$ An early functional change is believed by some ${ }^{12}$ to be upregulation of proliferation throughout the colon, not just at the site of the lesion. This study tested this hypothesis by assessing the CCPS in the human colon as assessed by mitosis counts in whole crypts for the macroscopically normal mucosa of both normal and tumour bearing colons.

\section{MEASUREMENT OF COLONIC CRYPT CELL}

PROLIFERATION

Dynamic methods of assessing proliferative activity within the colonic epithelium leading to a result in cells/crypt/hour or crypt cell production rate are more informative than static methods. The latter gives a measure of the number of cells in a given phase of the cell cycle in a moment in time-that is, crypt cell production state - and cannot therefore distinguish between changes in the proportion of cells in mitosis and changes in the duration of mitosis. The "gold standard" method in animal studies uses quantitation of arrested metaphases after systemic administration of a cytotoxic agent such as vincristine. ${ }^{7}$

This method has limited application in humans for obvious reasons although one such in vivo study was performed using systemic bromodeoxyuridine in patients undergoing colonic resection for colorectal carcinoma. ${ }^{5}$ Stathmokinetic techniques are used in human studies by employing in vitro culture of cell or tissue explants ${ }^{13}{ }^{14}$ which may not necessarily reflect the in vivo state. Cultured tissue is isolated from local influences of blood and tissue derived growth factors and exposure to luminal factors such as short chain fatty acids and bile acids. The more recent use of immunohistochemical staining of cell cycle related antigens by monoclonal antibodies such as PCNA and MIB1 have the advantage of being applicable to archival material but the results may be fixative dependan $\mathrm{t}^{1516}$ and there are fundamental problems of crypt architecture associated with assessing crypt sections that are overcome by the use of whole crypts. ${ }^{89}$

The present study provides evidence that whole crypt microdissection is a reliable method for assessing CCPS yielding consistent results for a given individual, since separate biopsies from one colonic site yielded similar 
results for a given individual (fig 2). In addition, estimates of CCPS seemed to be stable over time periods of several months. The results suggest that CCPS is independent of age over the range $20-70$ years, in contrast with the negative correlation with age reported by Deschner and colleagues. ${ }^{3}$ This lack of effect of age may not apply to subjects younger or older than those used in the present study (range 14-82 years). The finding suggests however that differences between the disease groups studied were not due to age even though patients with FAP were significantly younger than the other groups $(p=0.0001)$. There are other possible confounding factors for which it is difficult or impossible to control in human studies. All participants in this study were subjected to bowel preparation but the colectomy specimens were also devascularised from the moment the vascular pedicle was ligated to the time the mucosa was placed in fixative. However, since there was no overall difference in CCPS between specimens obtained at colonoscopy and those from colectomies, it seems unlikely that the sampling procedure was an important confounder. CCPS may also be subject to diurnal variation. ${ }^{4}$ All specimens in this study were obtained between 10.00 and 17.00 hours. At colonoscopy it is often difficult to be precise as to which part of the colon is being sampled but the combination of a single experienced endoscopist with deliberately broad division of sites into right, left, sigmoid colon, and rectum make a misallocation of biopsy site improbable.

CRYPT CELL PROLIFERATION AND COLORECTAL CANCER RISK

Controversy persists as to whether an increase in crypt cell proliferation in otherwise normal mucosa is an indication of early neoplastic activity. The finding of increased crypt cell proliferation in the apparently normal mucosa of individuals with colorectal cancer or FAP has been interpreted by some as an early field change leading to the normal mucosa $\rightarrow$ adenoma $\rightarrow$ carcinoma sequence. ${ }^{17}$ Others suggest this is a misinterpretation of patchy clonal proliferation leading to unicryptal adenomas. ${ }^{18}$ In addition, there has been failure to detect colonic mucosal hyperproliferation in mutation positive members of a family with HNPCC. ${ }^{19} 20$ Resolution of this controversy would have important consequences for the utility of crypt cell proliferation as a biomarker of cancer risk ${ }^{21}$ or as a surrogate endpoint in intervention studies. ${ }^{22}$ There is strong evidence, largely from animal studies, that crypt cell proliferation rate is readily modified by both the amount ${ }^{23}$ and composition of the diet ${ }^{24}$ and by diet related factors including bile acids ${ }^{25}$ and short chain fatty acids. ${ }^{26}$ An intestinal gradient in CCPR from the rectum to the caecum has been well established in animal studies in which a metaphase arrest agent (usually vincristine sulphate) can be administered in vivo. ${ }^{24}{ }^{27}$ It has been demonstrated that the caecum had a higher CCPR than the rest of the colon in the human large bowel. ${ }^{520} \mathrm{~A}$ similar trend has been shown using in vitro BrdU labelling in colectomy patients ${ }^{28}$ and $3 \mathrm{HTdR}$ labelling in adenoma patients. ${ }^{17}$ The findings reported here for both normal human colon and the "normal" mucosa of tumour bearing colon showed a higher CCPS in the more proximal colon (fig 4). This is difficult to reconcile with the site incidence of colorectal cancer (more common in the distal large bowel) if abnormal CCPS is to be regarded as a simple field change predisposing to the development of tumours.

Mucosal samples near to tumours $(<5 \mathrm{~cm})$ showed a small non-significant $(p=0.12)$ increased mitotic activity compared with distant samples $(>10 \mathrm{~cm})$ although Potten and colleagues in $1992^{5}$ found damping of the $\mathrm{BrdU}$ labelling index in samples from within $1 \mathrm{~cm}$ compared with those from more than $5 \mathrm{~cm}$ from the tumour edge. While it is feasible that a tumour may arise in an area of field change characterised by deregulation of cell proliferation, equally local autocrine trophic substances such as gastrin or enteroglucagon may be responsible. The observation that COX-2 is switched on in colonic tumours ${ }^{29}$ resulting in the production of eicosanoids and other substances which may increase proliferation and suppress apoptosis ${ }^{30}$ supports the view that alterations in crypt cell proliferation in tumour bearing colons may be a consequence rather than an aetiological factor in the development of neoplasia. Findings of altered crypt cell proliferation around a tumour are therefore best interpreted with caution.

A number of studies have found hyperproliferation of the normal colorectal mucosa in patients with sporadic adenomas ${ }^{12} 1731$ but this study was unable to detect any difference in CCPS between patients with sporadic adenomas and controls. When colorectal cancers were present, a significant increase in CCPS throughout the colon was observed $(p<0.01)$. Although this finding is thought by some to be part of the neoplastic process, ${ }^{12}$ it may be a reaction to unknown tumour related influences. ${ }^{18}$ However, this latter theory does not explain why this study found increased cellular proliferation in colons from which a cancer had previously been resected. Whatever the reason it can be considered to be a marker of increased neoplastic risk.

The most notable finding in this study was the increased CCPS in FAP colons compared not only with controls but also with colons bearing adenomas or cancer $(p<0.01)$. This observation may represent the earliest phase in the formation of unicryptal or oligocryptal adenoma or may indicate a diffuse hyperproliferative state. This study has not attempted to investigate why CCPS is raised in FAP. It is possible to speculate that the putative heterodimer protein product of a single mutated APC allele ${ }^{32}$ interacts abnormally with $\beta$-catenins resulting in uncontrolled growth. However, recent studies in two genetically engineered mouse models have shown that mutation in one APC allele had no effect on crypt cell proliferation in the macroscopically normal intestinal. ${ }^{33}{ }^{34}$ This suggests that inactivation of the second normal APC allele is 
required before the APC gene loses its function as "gatekeeper" of colonic epithelial cell proliferation. ${ }^{35}$

To summarise, whole crypt microdissection and mitosis counting is a reliable, reproducible, and robust technique for assessing CCPS in the human colon and this study confirms an increase in CCPS from the distal to the proximal colon. An increase in the CCPS in the macroscopically normal colonic mucosa was observed when a sporadic cancer was present. Similarly, the remarkable increase in CCPS in patients with an inherited mutation in the APC gene means that this finding indicates an increased likelihood of an FAP family member being a gene carrier. However, the precise relation of an increased CCPS to the neoplastic process remains uncertain.

Our thanks to all the staff of the endoscopy unit, Wansbeck Hospital, for their help and patience. Sarah Mills was funded by the Family Cancer Care Fund, Wansbeck District General Hospital, and a Northern Region Research and Development grant. Pamela Chapman is supported by ICRF. Additional funding was provided by the Cancer Research Campaign (project gran No SP 2323/0101)

1 Gerdes H, Gillin JS, Zimbalist E, et al. Expansion of the epithelial cell proliferative compartment and frequency of adenomatous polyps in the colon correlate with the strength of family history of colorectal cancer. Cancer Res 1993;53:279-82.

2 Lipkin M, Blattner WA, Gardner EJ, et al. Classification and risk assessment of individuals with familial polyposis, Gardner's syndrome, and familial non-polyposis colon cancer from $[3 \mathrm{H}]$ thymidine labeling patterns in colonic epithelial cells. Cancer Res 1984;44:4201-7.

3 Deschner EE, Godbold J, Lynch HT. Rectal cell proliferation in a group of young adults. Influence of age and tion in a group of young adults. Influence of age a

4 Buchi KN, Moore JG, Hrushesky WJM, et al. Circadian rhythm of cellular proliferation in the human rectal rhythm of cellular proliferation in the
mucosa. Gastroenterology 1991;101:410-15.

5 Potten CS, Kellett M, Rew DA, et al. Proliferation in human gastrointestinal epithelium using bromodeoxyuridine in vivo: data for different sites, proximity to a tumour, and polyposis coli. Gut 1992;33:524-9.

6 Lipkin M, Blattner WE, Fraumeni JF, et al. Tritiated thymidine labelling distribution as a marker for hereditary predisposition to colon cancer. Cancer Res 1983;43:1899-904.

7 Goodlad RA. Microdissection-based techniques for the determination of cell proliferation in gastrointestinal epithelium: application to animal and human studies. In: Celis JE, ed. Cell biology: A laboratory handbook. New York: Academic Press Inc., 1994:205-16.

8 Savidge TC, Walker-Smith JA, Phillips AD. Novel insights into human intestinal epithelial cell proliferation in health and disease using confocal microscopy. Gut 1995;36:369and

9 Goodlad RA, Levi S, Lee CY, et al. Morphometry and cell proliferation in endoscopic biopsies: Evaluation of a technique. Gastroenterology 1991;101:1235-41.

10 Leach FS, Nicolaides NC, Papadopoulos N, et al. Mutations of a mutS homolog in hereditary nonpolyposis colorectal cancer. Cell 1993;75:1215-25.

11 Bodmer W, Bishop T, Karran P. Genetic steps in colorectal cancer. Nat Genet 1994;6:217-19.

12 Ponz de Leon M, Roncucci L, Di Donato P, et al. Pattern of epithelial cell proliferation in colorectal mucosa of norma subjects and of patients with adenomatous polyps or cance of the large bowel. Cancer Res 1988;48:4121-6.
13 Kanemitsu T, Koike A, Yamamoto S. Study of the cell proliferation kinetics in ulcerative colitis, adenomatous polyps, and cancer. Cancer 1985;56:1094-8.

14 Roncucci L, Ponz de Leon M, Scalmati A, et al. The influence of age on colonic epithelial cell proliferation. Cancer 1988;62:2373-7.

15 Burford-Mason AP, MacKay AJ, Cummins M, et al. Detection of proliferating cell nuclear antigen in paraffinembedded specimens is dependant on preembedding tissue handling and fixation. Arch Pathol Lab Med 1994;118: handling

16 Hall PA, Woods AL. Immunohistochemical markers of celular proliferation: Acheivements, problems and prospects. Cell Tissue Kinet 1990;23:505-22.

17 Terpestra OT, van Blankenstein M, Dees J, et al. Abnormal pattern of cell proliferation in the entire colonic mucosa of patients with colon adenoma or cancer. Gastroenterology patients with colo

18 Jass JR. Evolution of hereditary bowel cancer. Mutat Res 1993;290:13-25

19 Jass JR, Ajioka Y, Radojkovic M, et al. Failure to detect colonic mucosal hyper proliferation in mutation positive members of a family with hereditary non-polyposis colorectal cancer. Histopathology 1997;30:201-7.

20 Green SE, Chapman P, Burn J, et al. Colonic epithelial cell proliferation in hereditary non-polyposis colorectal cancer. Gut 1998;43:85-92.

21 Preston-Martin S, Pike MC, Ross RK, et al. Increased cell division as a cause of human cancer. Cancer Res 1990;50:7415-21.

22 Cats A, Kleibeuker JH, van der Meer R, et al. Randomized, double-blinded, placebo-controlled intervention study with supplemental calcium in families with hereditary nonpoly-
posis colorectal cancer. $\mathcal{F}$ Natl Cancer Inst 1995;87:598posis 603.

23 Goodlad RA, Ratcliffe B, Fordham JP, et al. Does dietary fibre stimulate intestinal epithelial cell proliferation in germ free rats? Gut 1989;30:820-5.

24 Mathers JC, Kennard J. Gastrointestinal responses to oats consumption in young adult and elderly rats: digestion, large bowel fermentation and crypt cell proliferation rates. Br f Nutr 1993;70:567-84

25 Nagengast FM, Grubben MJAL, van Munster IP. Role of bile acids in colorectal carcinogenesis. Eur $\mathcal{f}$ Cancer 1995;31:1067-70

26 Sakata T. Stimulatory effect of short-chain fatty acids on epithelial cell proliferation in the rat intestine: a possible explanation for trophic effects of fermentable fibre, gut microbes and luminal trophic factors. Br F Nutr 1987;58: 95-103.

27 Sunter JP, Watson AJ, Wright NA, et al. Cell proliferation at different sites along the length of the rat colon. Virchows Arch B Cell Pathol 1979;32:75-87.

28 Hall C, Youngs D, Keighley MRB. Crypt cell production rates at various sites around the colon in Wistar rats and humans. Gut 1992;33:1528-31.

29 Eberhart CE, Coffey RJ, Radhika A, et al. Up-regulation of cyclooxygenase 2 gene expression in human colorectal adenomas and adenocarcinomas. Gastroenterology 1994; 107:1183-8.

30 Tsujii M, DuBois RN. Alterations in cellular adhesion and apoptosis in epithelial cells overexpressing prostaglandin endoperoxide synthase 2 . Cell 1995;83:493-501.

31 Anti M, Marra G, Armelao F. Rectal epithelial cell proliferation patterns as predictors of adenomatous colorectal polyp recurrence. Gut 1993;34:525-30

32 Polakis P. Mutations in the APC gene and their implications for protein structure and function. Curr Opin Genet Dev 1995;5:66-71.

33 Mathers JC, Kooshkghazi M, Coaker J, et al. Is crypt cell proliferation a reliable biomarker of intestinal cancer risk? Proc Nutr Soc 1998;j57:43A.

34 Wasan H, Mandir N, Park HS, et al. APC regulates crypt fission and not cell division in mice with multiple intestinal neoplasia (MIN). Proc Nutr Soc 1997;j57:45A.

35 Kinzler KW, Vogelstein B. Lessons from hereditary colorecal cancer. Cell 1996;87:159-70. 